\title{
Multi-Sensor Enabled Live Virtual Geographic Environment: A Way to the Implementation of Mirror World ${ }^{\dagger}$
}

\author{
Weitao Che 1,2,*, Hui Lin ${ }^{1,2}$ and Mingyuan $\mathrm{Hu}^{1,2}$ \\ 1 Shenzhen Research Institute, The Chinese University of Hong Kong, 518057 Shenzhen, China; \\ huilin@cuhk.edu.hk (H.L.); humingyuan@gmail.com (M.H.) \\ 2 Institute of Space and Earth Information Science, The Chinese University of Hong Kong, \\ Hong Kong, China \\ * Correspondence: cheweitao@link.cuhk.edu.hk \\ + Presented at the 5th International Symposium on Sensor Science (I3S 2017), Barcelona, Spain, \\ 27-29 September 2017.
}

Published: 30 November 2017

With the two cores of database and model-base, Virtual Geographic Environment (VGE) breaks the limitations of the traditional Geographic Information System (GIS), which provides new support to the study and understanding of our world. VGE is proposed as a new generation of geographic language that is characterized by "feeling it in person, knowing it beyond reality". Unlike traditional data-centered GIS, VGE is a human-centered environment that represents and simulates both physical and human environments.

In the past, the data that fueled GIS/VGE was typically created to represent the state of the geoscape at a specific moment in time ("historic" or "current"; or "future" to represent a future modeled state). While this kind of data has been proven to be very valuable for numerous applications and analyses, even the "current" snapshot quickly falls out of sync with the real world. The fast-paced, constantly changing world makes the "current" snapshot outdated as soon as it is created. Fortunately, advances in sensor technology are revolutionizing the way in which geospatial information is collected and analyzed. For example, cameras and GPS sensors have the ability to provide continuous streams of geospatially-rich information.

In this topic, the emergence of integrating multi-sensor data and VGE is illustrated firstly. For this, multi-sensor enabled Live VGE is proposed as a new branch of VGE study to describe the everchanging world, which is a way to implement the Mirror World. Mirror World is the virtual environment that is parallel the real world to facilitate its understanding and engagement through tools for interpreting massive data streams and shared decision-making at varying temporal and spatial scales. In our multi-sensor enabled Live VGE, multiple kinds of sensors such as GPS, in-situ sensors, visual sensors, etc., are used to incorporate the temporal aspect and real-time information into the geographic environment. A framework of Live VGE is given based on the proposed concept, in which multiple sensor data are geo-coded and integrated with the 3D VGE platform. A customized geosensor network service for Live VGE is provided to manage all the sensors and format the sensor data stream. After that, a new reconstruction method for these sensor data, especially the mobile sensing data, is mentioned to meet the demands of real-time visualization and simulation. Finally, environmental monitoring and simulation based case studies and experiments are also presented.

Acknowledgments: The work was supported by National Natural Science Foundation of China (41401459) and National Key Basic Research Program of China (2015CB954103).

(C) 2017 by the authors. Licensee MDPI, Basel, Switzerland. This article is an open access article distributed under the terms and conditions of the Creative Commons Attribution (CC BY) license (http://creativecommons.org/licenses/by/4.0/). 